\title{
Non-destructive mapping of long-range dislocation strain fields in an epitaxial complex metal oxide
}

Simons, Hugh; Jakobsen, Anders Clemen; Ahl, Sonja Rosenlund; Poulsen, Henning Friis; Pantleon, Wolfgang; Chu, Ying-Hao; Detlefs, Carsten; Valanoor, Nagarajan

\section{Published in:}

Nano Letters

Link to article, DOI:

10.1021/acs.nanolett.8b03839

Publication date:

2019

Document Version

Publisher's PDF, also known as Version of record

Link back to DTU Orbit

Citation (APA):

Simons, H., Jakobsen, A. C., Ahl, S. R., Poulsen, H. F., Pantleon, W., Chu, Y-H., Detlefs, C., \& Valanoor, N. (2019). Non-destructive mapping of long-range dislocation strain fields in an epitaxial complex metal oxide. Nano Letters, 19(3), 1445-1450. https://doi.org/10.1021/acs.nanolett.8b03839

\section{General rights}

Copyright and moral rights for the publications made accessible in the public portal are retained by the authors and/or other copyright owners and it is a condition of accessing publications that users recognise and abide by the legal requirements associated with these rights.

- Users may download and print one copy of any publication from the public portal for the purpose of private study or research.

- You may not further distribute the material or use it for any profit-making activity or commercial gain

- You may freely distribute the URL identifying the publication in the public portal 


\title{
Nondestructive Mapping of Long-Range Dislocation Strain Fields in an Epitaxial Complex Metal Oxide
}

\author{
Hugh Simons, ${ }^{*} \dagger$ Anders Clemen Jakobsen, ${ }^{\dagger}$ Sonja Rosenlund Ahl, ${ }^{\dagger}$ Henning Friis Poulsen, ${ }^{\dagger}$ \\ Wolfgang Pantleon, ${ }^{\ddagger}$ Ying-Hao Chu, ${ }^{\S ®}$ Carsten Detlefs, ${ }^{\|}$and Nagarajan Valanoor ${ }^{\perp}$ \\ ${ }^{\dagger}$ Department of Physics, Technical University of Denmark, Kgs. Lyngby 2800, Denmark \\ ${ }^{\ddagger}$ Department of Mechanical Engineering, Technical University of Denmark, Kgs. Lyngby 2800, Denmark \\ ${ }^{\S}$ Department of Materials Science \& Engineering, National Chiao Tung University, Hsinchu 30010, Taiwan \\ "European Synchrotron Radiation Facility, 71 Avenue des Martyrs, 38043 Grenoble Cedex 9 France \\ ${ }^{\perp}$ School of Materials Science \& Engineering, University of New South Wales, Kensington 2052, Australia
}

ABSTRACT: The misfit dislocations formed at heteroepitaxial interfaces create long-ranging strain fields in addition to the epitaxial strain. For systems with strong lattice coupling, such as ferroic oxides, this results in unpredictable and potentially debilitating functionality and device performance. In this work, we use dark-field $\mathrm{X}$-ray microscopy to map the lattice distortions around misfit dislocations in an epitaxial film of bismuth ferrite $\left(\mathrm{BiFeO}_{3}\right)$, a wellknown multiferroic. We demonstrate the ability to precisely quantify weak, long-ranging strain fields and their associated symmetry lowering without modifying the mechanical state of the film. We isolate the screw and edge components of the individual dislocations and show how they result in weak charge heterogeneities via flexoelectric coupling. We show that even systems with small lattice mismatches and additional mechanisms of stress relief (such as mechanical twinning) may still give rise to measurable charge and strain heterogeneities that extend over mesoscopic length scales. This sets more stringent physical limitations on device size, dislocation density, and the achievable degree of lattice mismatch in epitaxial systems.

KEYWORDS: Ferroelectric, dislocation, BiFeO3, strain, microscopy, diffraction

A semiconductor and complex metal oxide nanoelectronics employ increasingly aggressive strain-engineering strategies, a comprehensive understanding of the functional consequences of atomic-scale defects has never been more important. ${ }^{1}$ In particular, dislocations undermine straininduced phenomena by altering local chemistry at the dislocation core ${ }^{2}$ and relaxing imposed epitaxial strain; ${ }^{3}$ even single dislocations create long-range strain fields that significantly compromise the electronic structure ${ }^{4}$ and physio-chemical nature of the material. ${ }^{5}$

Comprehensively understanding the collective influence of defects such as dislocations requires precise, quantitative, and spatially resolved measurements of the lattice distortions they create. For many years, the main approaches were dark-field electron microscopy ${ }^{6}$ and X-ray topography, ${ }^{7}$ in which Bragg diffraction provided great sensitivity to lattice distortions through the intensity fringes surrounding the dislocation(s). These fringes often persist over several micrometers from the dislocation core, demonstrating the presence of elastic interactions over mesoscopic distances. However, the complexity of the dynamical scattering process responsible for the fringes made quantitative and unambiguous determination of the strain fields and lattice symmetry challenging, if not impossible. The more recent development of atomic resolution scanning transmission electron microscopy (STEM) now enables strain and symmetry to be mapped directly from the atomic displacements. ${ }^{8}$ This approach is intuitive and direct; however, (1) the field-of-view is restricted to only tens of unit cells (i.e., nanoscale) around the dislocation core; (2) the strain resolution $\left(\sim 10^{-3}\right)$ is insufficient to detect elastic strain fields slightly beyond the dislocation cores; ${ }^{9}$ and (3) samples must be thin $(20-30 \mathrm{~nm})$ cross sections, meaning the lateral (i.e., in-plane) mechanical constraints are very different from those of the macroscopic film.

The capability to measure weak strain fields (i.e., well below $10^{-3}$ ) over mesoscopic scales without perturbing the mechanical state of the material (i.e., nondestructively) has recently become available with the development of dark-field $\mathrm{X}$-ray microscopy. ${ }^{10}$ The technique combines sub-100 nm spatial resolution with a $400 \times 200 \mu \mathrm{m}^{2}$ field of view and sensitivity to small structural distortions of the order of $10^{-5} .11$ Like its electron-based analogue, dark-field X-ray microscopy uses an X-ray objective lens in the Bragg-diffracted X-ray beam to create a magnified image of the sample corresponding to a restricted and known volume in reciprocal space. ${ }^{12}$ It requires similar instrumentation to X-ray diffraction topography ${ }^{13}$ and

Received: September 22, 2018

Revised: January 31, 2019

Published: February 6, 2019 


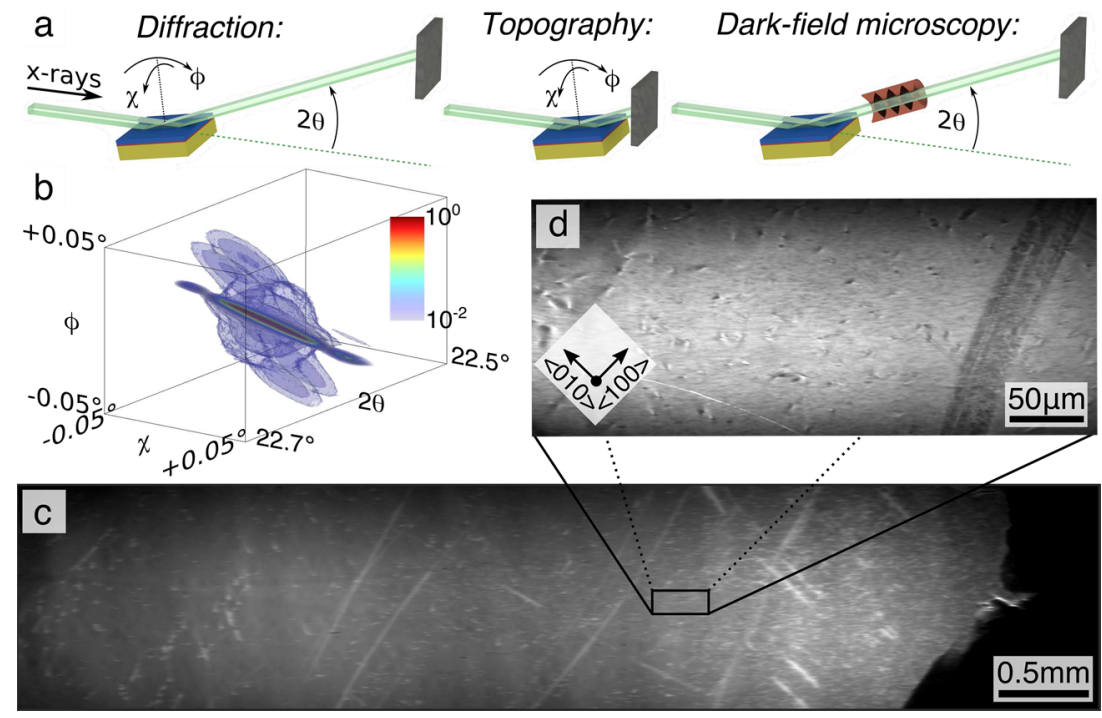

Figure 1. Multiscale diffraction measurements on the $\mathrm{BiFeO}_{3} / \mathrm{SrRuO}_{3} / \mathrm{DyScO}_{3}$ film. The three types of measurement (a) comprised 3D reciprocal space maps (i.e., diffraction) using a $2 \mathrm{D}$ detector with moderate resolution $(54 \mu \mathrm{m})$ far from the sample, large field-of-view maps of the dislocation distribution (i.e., X-ray topography) using a high-resolution detector close to the sample, and high-resolution orientation/inclination and elastic strain maps (i.e., dark-field X-ray microscopy) using a magnifying lens between the sample and a high-resolution detector. The resulting diffracted intensity distribution around the 002 reflection (b) shows peak broadening from dislocations and lobes from the ferroelectric domain structure (note that intensity is shown on a logarithmic scale). X-ray topography map (c) showing the coarse-scale distribution of the dislocations, which can be seen in individual detail in (d) the dark-field X-ray microscopy image. All diffraction measurements were carried out on the 002 reflection with an X-ray energy of $17 \mathrm{keV}$ at ID06 of the European Synchrotron Radiation Facility.
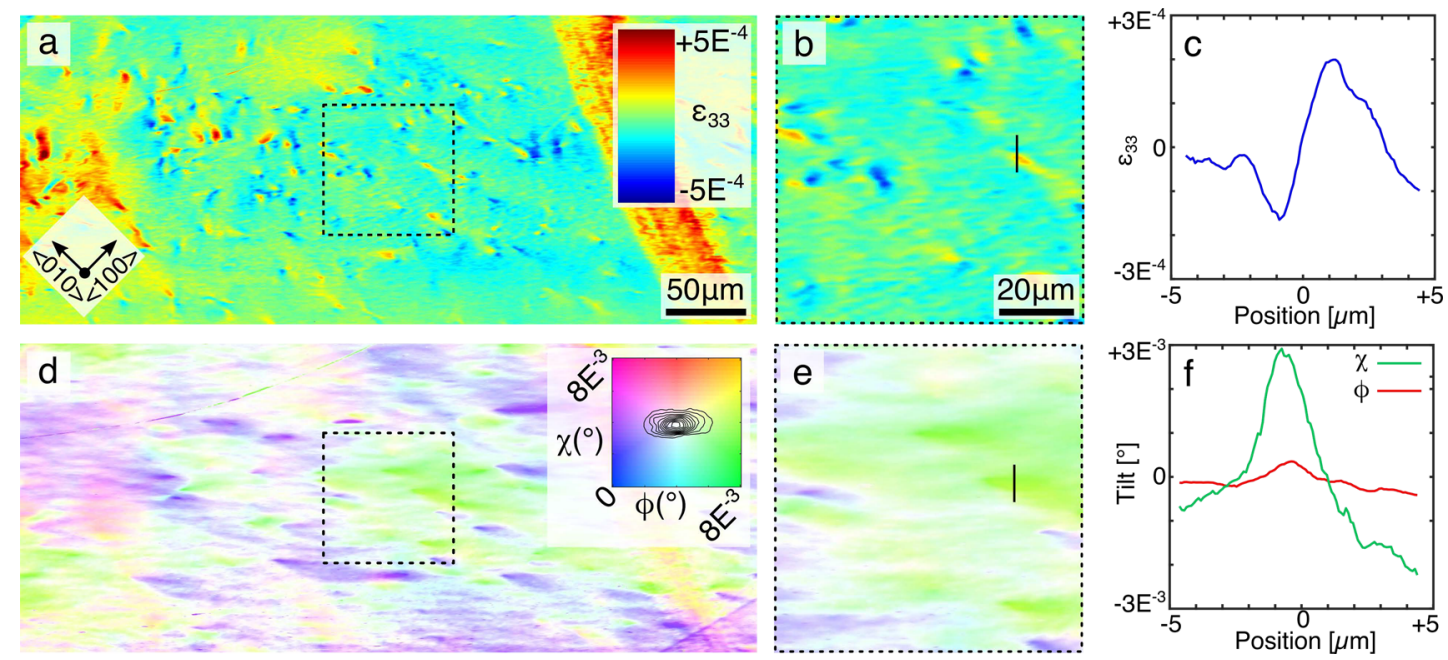

Figure 2. Dark-field microscopy maps of the $\mathrm{BiFeO}_{3}$ film around the 002 Bragg reflection. (a) Out-of-plane lattice strain $\varepsilon_{33}$, (b) close-up of the strain fields due to individual dislocations, and (c) strain profile through the black line in (b). The local lattice plane inclination of the same region (d) and corresponding close-up (e) are colored according to the key (right), which is superimposed with a contour map of the diffraction intensity of the entire map. Corresponding profiles of the lattice tilt components $(\chi, \phi)$ are additionally shown in (f). Note that the red region to the right of (a) is a scratch in the film surface and is not considered in this (or subsequent) analysis.

X-ray reciprocal space mapping ${ }^{14}$ (Figure 1a), allowing it to be carried out in conjunction with these techniques in quick succession to intuitively create a multiscale picture of the structure and topology (Figure $1 \mathrm{~b}-\mathrm{d}$ ). Crucially, this combination of a large sample size, nondestructive measurements, and exceptional angular resolution presents opportunities for new types of measurement not previously possible.

Here, we apply dark-field X-ray microscopy to quantify the long-range structural distortion fields (elastic axial strain and lattice plane inclination) around individual dislocations in an epitaxial film of bismuth ferrite $\left(\mathrm{BiFeO}_{3}\right) . \mathrm{BiFeO}_{3}$ is an important model system for oxide nanoelectronics and a single-phase room-temperature multiferroic exhibiting three strain-coupled functional responses, namely, ferroelectricity, ferroelasticity, and antiferromagnetism. Furthermore, $\mathrm{BiFeO}_{3}$ exhibits defect-driven phenomena (e.g., memristivity, ${ }^{15}$ domain-wall conductivity, ${ }^{16}$ and photoelectricity ${ }^{17}$ ) believed to be susceptible to heterogeneous strain. Lowering the nominal symmetry through strain engineering and heteroepitaxy is a well-known way to induce new or radically modified functionality, ${ }^{18}$ but understanding the physical consequences of these symmetry-breaking distortions first requires identifying their origin and quantifying their effect on the local structure. The ability to provide a clear description of the 

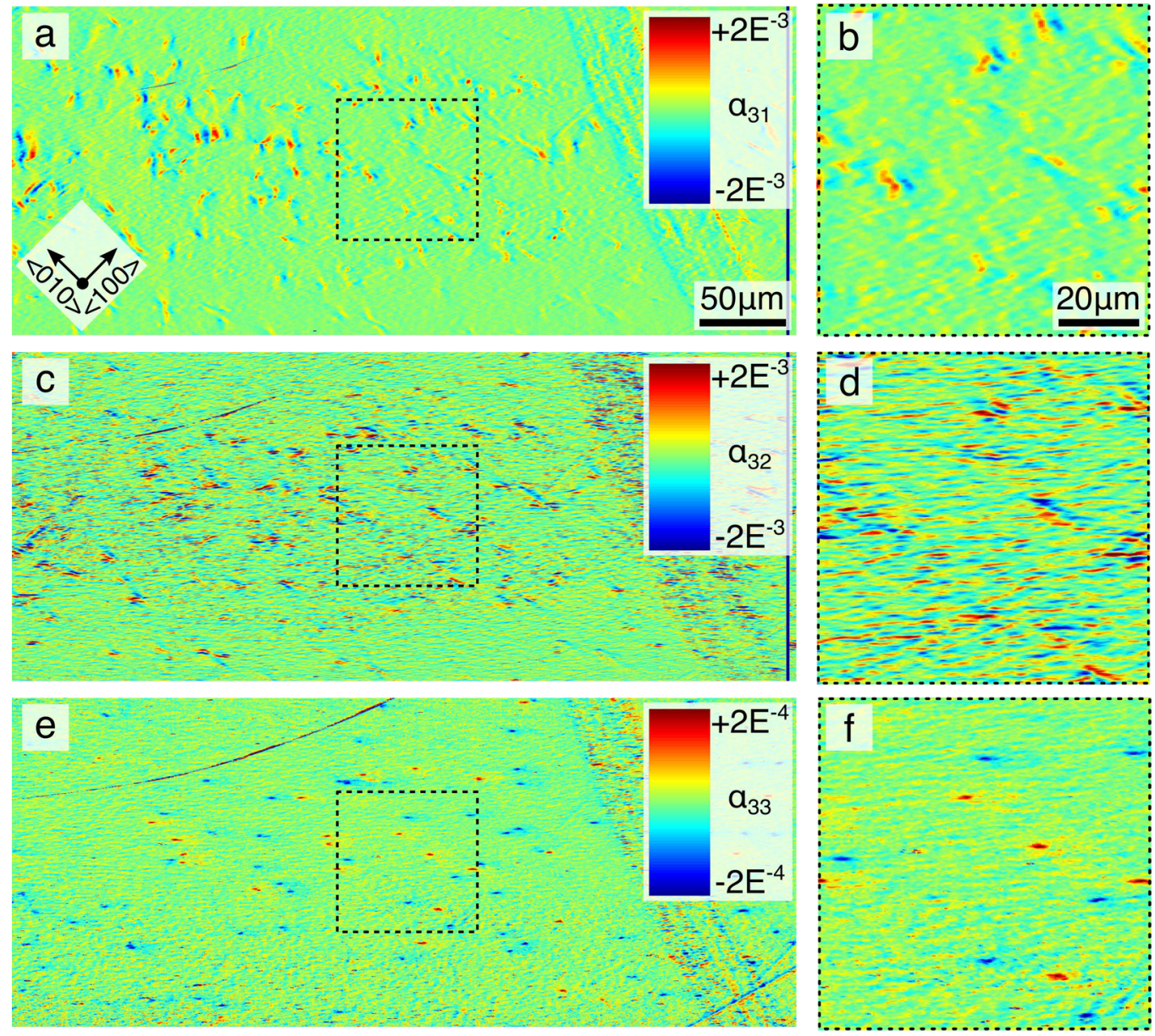

Figure 3. Maps of the resolvable components of the dislocation density tensor. The $\alpha_{31}(\mathrm{a}, \mathrm{b})$ and $\alpha_{32}$ (c, d) components reflect edge dislocation densities, while the $\alpha_{33}(\mathrm{e}, \mathrm{f})$ component represents screw dislocation density. The sign of the density corresponds to the sign of the edge components or the chirality of the screw dislocations. Correlated edge and screw densities are highlighted by the diagonal box on panels (b), (d), and (f). Note that $\alpha_{i k}$ has undergone a coordinate transformation corresponding to a rotation by $45^{\circ}$ around the film normal such that the axis $x_{1}$ and $x_{2}$ for $\alpha_{31}$ and $\alpha_{32}$ are parallel to the [100] and [010] directions, respectively.

consequences of dislocations in $\mathrm{BiFeO}_{3}$, and in particular their long-range strain fields, would catalyze a new understanding of how these dislocations relax the epitaxial strain, as well as how long-range strains influence critical parameters in complex metal oxides.

The sample used here was a $120 \mathrm{~nm}$ thick film of $<001>$ oriented $\mathrm{BiFeO}_{3}$, grown via pulsed laser deposition on a $\mathrm{SrRuO}_{3}$ electrode layer and $<110>$-oriented $\mathrm{DyScO}_{3}$ single crystalline substrate (note all Miller indices are indexed as $P m \overline{3} m)$. The film had highly periodic, $<111>$-polarized stripe domains with an average width of $0.2 \mu \mathrm{m}$ and domain walls along [110] directions (see Methods and ref 19 for details). As in other $<001>$-oriented perovskites, dislocations are expected to have Burgers vectors with out-of-plane components, such as along [101] or [011] directions. ${ }^{20-23}$ The X-ray measurements carried out for this work utilized the specular 002 reflection, meaning that structural contrast arose only from out-of-plane atomic displacements. This implies that the lattice distortions from $<111>$-polarized domains and 110-oriented domain walls should be weak in comparison to other sources of symmetrybreaking distortions, such as dislocations. ${ }^{24}$ Shown in Figure $1 \mathrm{~b}$, the X-ray reciprocal space map around the 002 reflection of the $\mathrm{BiFeO}_{3}$ shows clear evidence of these symmetry-breaking lattice distortions in the film, i.e., four lobes of increased intensity at small angles from the main peak classically associated with lattice rotations across the ferroelastic $<110>$-oriented domain walls ${ }^{25}$ and diffuse intensity surrounding the main peak attributable to symmetry breaking by dislocations $^{26}$ or non-Ising domain walls. ${ }^{27}$ Finely mottled intensity contrast in the X-ray topography image (Figure 1c) suggests that local structural defects are distributed across the entire film. Using dark-field X-ray microscopy to magnify this mottled intensity contrast (Figure $1 \mathrm{~d}$ ) reveals no evidence of domain walls (as expected from the 002 reflection) but sharp points of intensity variation consistent with individual dislocations. $^{26}$

Figure 2 shows maps of the axial strain $\varepsilon_{33}$ and the lattice plane inclination $(\chi, \phi$; see Figure 1a for schematic) for a 200 $\times 400 \mu \mathrm{m}^{2}$ region of interest near the center of the sample obtained by dark-field X-ray microscopy. The strain and inclination variations are correlated, as elongated lobes of positive and negative strain in (b) correspond to sharp changes in inclination in (d). Note that the strain lobes in Figure $2 b$ and $2 \mathrm{c}$ typically extend $1-8 \mu \mathrm{m}$ in [100] and [010] directions and in some cases mutually superimpose.

The quantitative measurement of lattice distortions over such a large spatial range, i.e., several micrometers, is a key result. In this manner, we can quantify the long-ranging strain fields and unambiguously demonstrate that the observed distortions locally break the nominal rhombohedral symmetry 

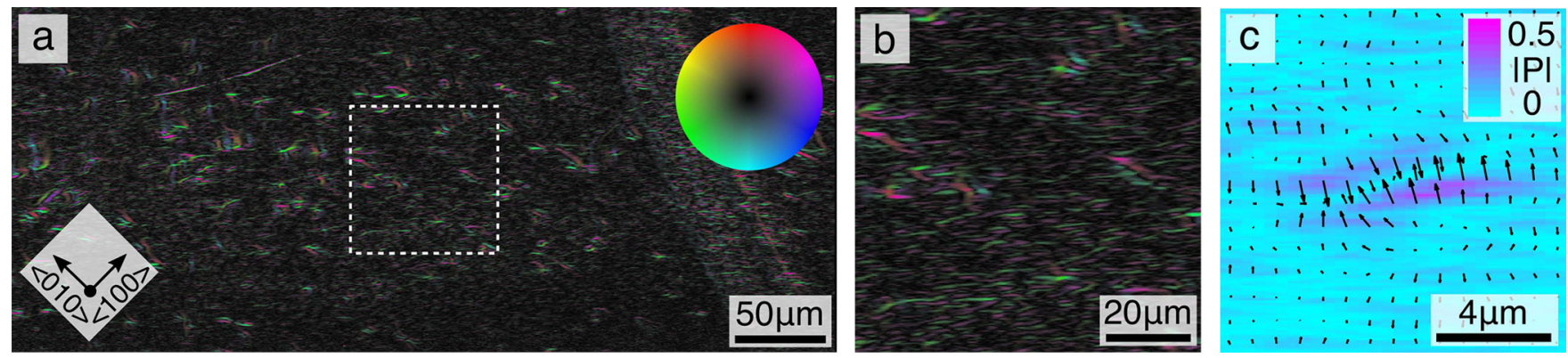

Figure 4. Flexoelectric polarization contributions. Magnitude and orientation over the $\mathrm{BiFeO}_{3}$ film surface and close-up (a, b), where orientation and magnitude of the polarization component are given by hue and lightness, respectively. The perimeter of the colorwheel corresponds to a polarization magnitude of $0.40 \mu \mathrm{C} / \mathrm{cm}^{2}$, while the maximum value in the image was $1.42 \mu \mathrm{C} / \mathrm{cm}^{2}$. A further close-up of the polarization augmentation around a single dislocation (c) which shows head-to-head polarization toward the core, resulting in a net electrical charge at the dislocation core. The polarization magnitude in (c) is given in units of $\mu \mathrm{C} / \mathrm{cm}^{2}$ and is calculated assuming a flexoelectric coefficient of $\mu=1.4 \mu \mathrm{C} /$ $\mathrm{m}$ (from ref 39).

(space group $R 3 c$ ). The quantitative nature of the lattice strain and inclination maps allow the type, size, positions, and directions of the individual dislocations to be determined. As we probe the $<001>$-oriented $\mathrm{BiFeO}_{3}$ film with the 002 scattering vector, $Q_{002}$, the measurements describe the lattice perturbations generated by dislocations with parallel, out-ofplane components to their Burgers vector. Dislocations with both edge and screw characteristics contribute to these distortions, and can be described by the local dislocation density tensor ${ }^{28} \alpha_{i k}=\sum_{t=1}^{N} b_{i}^{t} l_{k}^{t} \rho^{t}=-\epsilon_{k l j} \frac{\partial \beta_{i j}}{\partial x_{l}}$ originating from the individual dislocation densities $\rho^{t}$ of all different dislocation types $t$, each characterized by their Burgers vector $b_{i}^{t}$ and line vector $l_{k}^{t}$. Alternatively, the components of dislocation density tensor can be found as gradients of the elastic distortion tensor $\beta_{i j}$ with respect to the spatial dimension $x_{l}$ via the Levi-Civita permutation symbol $\epsilon_{k l j}{ }^{29}$ In turn, the elastic distortion tensor $\beta_{i j}$ is linked to the elastic strain tensor $\varepsilon_{i j}$ and the lattice rotations. From the nine components of the dislocation tensor $\alpha_{i k}$, three components are resolvable from the maps of $\varepsilon_{33}$ (i.e., out-of-plane) strain and the $\phi$ and $\chi$ lattice inclinations: ${ }^{30}$

$$
\begin{aligned}
& \alpha_{31}=-\frac{\Delta \beta_{33}}{\Delta x_{2}}=-\frac{\Delta \varepsilon_{33}}{\Delta y} \\
& \alpha_{32}=\frac{\Delta \beta_{33}}{\Delta x_{1}}=\frac{\Delta \varepsilon_{33}}{\Delta x} \\
& \alpha_{33}=-\left(\frac{\Delta \beta_{32}}{\Delta x_{1}}-\frac{\Delta \beta_{31}}{\Delta x_{2}}\right)=-\left(\frac{\Delta \varphi}{\Delta y}+\frac{\Delta \chi}{\Delta x}\right)
\end{aligned}
$$

The differences in the symmetry of the elastic distortion fields generated by dislocations of different character (i.e., screw or edge) mean that different components of $\alpha_{i k}$ represent dislocation densities of different character, i.e., the $\alpha_{31}$ and $\alpha_{32}$ components correspond to the local density of edge dislocations, while the $\alpha_{33}$ component corresponds to the local density of screw dislocations. ${ }^{30}$

In Figure 3, maps of $\alpha_{31}, \alpha_{32}$, and $\alpha_{33}$ show the densities of dislocations in the $\mathrm{BiFeO}_{3}$ film with edge and screw character and an out-of-plane component of the Burgers vector. The $\alpha_{31}$ and $\alpha_{32}$ maps reveal similarly elongated features as the strain maps and profiles in Figure 2, suggesting edge dislocation lines spanning over several micrometers. The $\alpha_{33}$ map, however, shows points of large positive or negative dislocation density, predominantly in pairs. Their magnitude, which is typically in the order of $2 \times 10^{-4} \mathrm{~nm}^{-1}$, is comparable to the estimated value for a single screw dislocation within a pixel $(b / \Delta x \Delta y=$ $2.5 \times 10^{-4} \mathrm{~nm}^{-1}$ for a length of the Burgers vector $b$ of the order of the lattice parameter, $a$ ), suggesting each pixel contains a single threading dislocation with a partial screw character. In addition, the difference in the chirality between two connecting threading dislocation is reflected in the opposite sign of the dislocation density $\alpha_{33}$.

A clear picture now emerges of the mesoscale strain fields generated by dislocations. In several places, the dislocation density maps conform to the classical perception for a misfit dislocation in which an edge dislocation with a line direction parallel to the film-substrate interface is terminated by a pair of right- and left-handed screw dislocations, albeit here with out-of-plane components of the Burgers vectors rather than the common in-plane component. In Figure 3, such connecting edge dislocations are suggested by the elongated features along [100] and [010] directions in (3b and $3 \mathrm{~d})$ traversing between $\alpha_{33}$ components of opposite sign (3f), i.e., screws of the same Burgers vector with opposite chirality. We note that, in principle, it is possible to determine the sign of the Burgers vector of the misfit dislocation from the chiralities of a given pair of screw components. This information would be particularly valuable in assessing the effectiveness of the misfit dislocations at relaxing the epitaxial strain. However, the relevant component of the Burgers vector for this relaxation will be in-plane and is not accessible by imaging the out-ofplane lattice distortions as per the measurement configuration used here. Only imaging a Bragg reflection with an in-plane component (e.g., 110 or 111) would reveal the complementary relaxation mechanisms of both the ferroelastic stripe domains and the misfit dislocations. In fact, the faint periodic contrast in the images is an artifact arising from aliasing of small lattice rotations from ferroelectric domain walls, which have a similar periodicity to the spatial resolution of the instrument. The interactions of these domains directly with the misfit dislocations cannot be clearly established here; however, piezoresponse force microscopy (PFM) images of this sample ${ }^{19}$ show domains terminating at line defects with similar spacing and attributes as the dislocation described here.

Having established that the long-range lattice distortion fields observed here (and shown in Figure 2) originate from dislocations (as revealed by their densities in Figure 3), we now consider the impact of these mesoscale strain fields on the 
local ferroelectric properties. Because dislocations break the $R 3 c$ crystal symmetry, so too do their ensuing strain fields. This has two implications: (1) extrinsic strains that cant the polarization vector may permit polarization rotation mechanisms, which are known to impact dynamic dielectric and piezoelectric properties significantly, ${ }^{31}$ and (2), the distortion fields can directly contribute to the static polarization through the flexoelectric effect. ${ }^{32}$

Flexoelectricity is a linear coupling between polarization and the elastic distortion gradient via the flexoelectric coupling coefficient $\mu: P_{l}=\mu_{l i j k} \frac{\partial \beta_{i j}}{\partial x_{k}}$. $^{32}$ The effect occurs in all insulating crystals and is shown to influence a range of phenomena including localized photoelectricity, ${ }^{17}$ polarization rotation at domain walls, ${ }^{33}$ and measurable piezoelectricity above the Curie temperature, $T_{\mathrm{C}}{ }^{34}$ To date, most studies of flexoelectricity in ferroic materials have focused on the strain fields generated by domain walls, which decay exponentially with distance from the wall. ${ }^{35}$ In comparison, however, dislocations have a slower, hyperbolic decay of the distortion fields ${ }^{36}$ that should result in flexoelectric effects over much longer distances than that of domain walls. Surprisingly, the flexoelectric effect due to dislocations in a ferroelectric matrix has not garnered the same level of interest as domain walls in ferroelectric materials, despite the long-ranging strain gradients being significantly larger than those associated with ferroelastic domain walls.

Figure 4 shows this long-range flexoelectric polarization contributions clearly; the in-plane flexoelectric polarization $P_{i}$ (i.e., its magnitude and direction) is derived from direct measurements of the out-of-plane strain gradient assuming $\mu_{133 k}=\mu \delta_{l k}$ (note the formulation of the flexoelectric polarization in terms of elastic strain gradients ${ }^{37,38}$ is only justified for compatible elastic distortion fields; as the distortion field around dislocation cores itself is locally incompatible, the above formulation based on the elastic distortion tensor $\beta_{i j}$ has to be used). The magnitude of the polarization $|P|$ is enhanced in the vicinity of the edge dislocations detected in Figure 3 and remains measurable up to a few micrometers from the perceived dislocation core (Figure $4 \mathrm{~b})$. Furthermore, the flexoelectrically induced polarization is canted toward the dislocation core in a head-to-head arrangement, resulting in a net charge (Figure 4c). Similarly, dislocations whose edge components have opposite signs result in tail-to-tail configurations and a localized net charge of opposite sign. In the absence of an accepted theoretical value of the flexoelectric coefficient for rhombohedral $\mathrm{BiFeO}_{3}$, our calculations of the magnitude of the flexoelectric polarization used the experimentally determined value for M1 phase $\mathrm{BiFeO}_{3}$ of $\mu=1.4 \mu \mathrm{C} / \mathrm{m}$. ${ }^{39}$ This would suggest long-ranging polarization contributions up to $1.4 \mathrm{C} / \mathrm{cm}^{2}$; however, we note that the real value may be lower, as the experimentally determined flexoelectric coefficient is approximately 3 orders of magnitude larger than the accepted values for other oxide perovskites (e.g., $\mathrm{SrTiO}_{3}$ ). ${ }^{40}$ It is therefore difficult to precisely ascertain the significance of this flexoelectric contribution. However, it is conceivable that even the presence of very small charge fluctuations across the film will give rise to screening behavior, either by the domain structure via curved or otherwise charged domain walls, or by free carriers and vacancies, which should be abundant in $\mathrm{BiFeO}_{3}$. This may then have indirect influences upon the local depolarization behavior $^{41}$ and defect chemistry. ${ }^{42}$
These results demonstrate dark-field X-ray microscopy as a new probe for quantitatively and nondestructively mapping of small structural distortions over mesoscale distances. Our results show how strain fields of dislocations also affect the mesoscale structure and polarization, with distinct deviations from the nominal lattice symmetry across the majority of the sample and heterogeneous polarization perturbations due to flexoelectricity. Combined with atomic resolution microscopy studies of the chemical and functional properties in the immediate vicinity of the cores, we are now in a position to genuinely quantify the lattice-functional response across four orders of magnitude in length. This is a major step toward the development of computational materials engineering approaches $^{43}$ through the validation of multiscale models that predict how individual defects influence macroscopic structure and properties. The mesoscale structural and functional perturbations we measure here are small; however, given the sensitivity of nanoelectronic devices to small changes in electronic structure and local crystallography, it is plausible that dislocations, and our ability to fully characterize them, will have appreciable consequences across a broad spectrum of materials and devices.

Methods. Sample Preparation. A detailed description of the sample manufacture is provided in ref 19.

$X$-Ray Configuration. All X-ray measurements were carried out on the hard X-ray microscope at the beamline ID06 of the European Synchrotron Radiation Facility (ESRF). An X-ray energy of $17 \mathrm{keV}$ was used, with a bandwidth of $10^{-5}$ (rms) selected by a Si 111 double monochromator. A Be transfocator $30 \mathrm{~m}$ from the undulator prefocused the X-rays onto the sample, which was located $10 \mathrm{~m}$ further downstream. The beam profile at the focal point was approximately Gaussian, with dimensions of $200 \mu \mathrm{m}$ (horizontal) $\times 50 \mu \mathrm{m}$ (vertical), confirmed by direct imaging with a CCD camera. The sample was positioned such that the beam entered the sample at the approximate center of the $5 \times 5 \mathrm{~mm}^{2}$ face when tilted in the Bragg condition for the 002 reflection (i.e., $\theta+\phi=11.3^{\circ}$ and $\left.2 \theta=22.6^{\circ}\right)$. Indirect $2 \mathrm{D}$ detectors composed of a scintillator, visible optics, and CCD camera were used.

Reciprocal Space Mapping. Reciprocal space maps used a Basler CCD camera, $12 \mathrm{~mm} \mathrm{f} / 1.4$ machine vision optics, and a plastic scintillator with a $ø 50 \mathrm{~mm}$ circular field of view with 54 $\mu \mathrm{m}$ pixels. This field of view acquires approximately $2.22^{\circ}$ in $\chi$ and $0.55^{\circ}$ in $2 \theta$ in each image. Exposures of $1 \mathrm{~s}$ were recorded while incrementally tilting the $\phi$ angle through $0.6^{\circ}$ in 500 steps. These image series were then computationally interpolated into linear $\phi-\chi-2 \theta$ space.

$X$-Ray Topography. These measurements used a FReLoN CCD detector with a $2048 \times 2048$ array of $0.622 \times 0.622 \mu \mathrm{m}$ pixels positioned $5 \mathrm{~mm}$ from the sample. A series of 500 images were measured with $0.3 \mathrm{~s}$ exposures while tilting $\phi$ from $-0.1^{\circ}$ to $0.1^{\circ}$.

Dark-Field X-Ray Microscopy. Dark-field X-ray microscopy measurements required inserting an X-ray objective lens and a higher-resolution CCD camera into the diffracted beam path. The X-ray objective comprised a Be compound refractive lens with a focal length of $25 \mathrm{~cm}$, which gave an X-ray magnification of $15.9 \mathrm{x}$ at a numerical aperture of $5 \times 10^{-4}$. The FReLoN CCD camera used a LAG scintillator and a 10x microscope objective, yielding an effective pixel size of $1.4 \mu \mathrm{m}$. Combined, the dark-field microscope had a spatial resolution of approximately $82 \mathrm{~nm}$ (vertical) $\times 420 \mathrm{~nm}$ (horizontal). Image acquisition consisted of recording a $3 \mathrm{~s}$ exposure as 
the sample was progressively tilted through $\chi$ and $\phi$, and the objective and were detector tilted through $2 \theta$ in 20 steps over $1^{\circ}$ per direction. Details can be found in refs 10 and 12 .

\section{AUTHOR INFORMATION}

\section{Corresponding Author}

*E-mail: husimo@fysik.dtu.dk.

\section{ORCID $\odot$}

Hugh Simons: 0000-0002-3093-9241

Ying-Hao Chu: 0000-0002-3435-9084

Nagarajan Valanoor: 0000-0003-2534-5868

\section{Author Contributions}

Y.H.C. grew the sample. H.S. carried out the measurement with assistance from A.C.J. and S.R.A. H.S. analyzed the data and then interpreted it with N.V. and W.P. H.S. wrote the manuscript with input from N.V., W.P., C.D., and H.F.P.

\section{Notes}

The authors declare no competing financial interest.

\section{ACKNOWLEDGMENTS}

We are grateful to the European Synchrotron Radiation Facility for providing beamtime on ID06 and to Danscatt for funding the associated travel. H.S. acknowledges financial support from a DFF-FTP individual postdoctoral grant. H.F.P. acknowledges an ERC advance grant (d-TXM). Finally, we would like to thank the two anonymous reviews for their insightful and constructive feedback.

\section{REFERENCES}

(1) Martin, L. W.; Rappe, A. M. Nat. Rev. Mater. 2017, 2, 16087.

(2) Arredondo, M.; Ramasse, Q. M.; Weyland, M.; Mahjoub, R.; Vrejoiu, I.; Hesse, D.; Browning, N. D.; Alexe, M.; Munroe, P.; Nagarajan, V. Adv. Mater. 2010, 22, 2430-2434.

(3) Speck, J. S.; Daykin, A. C.; Seifert, A.; Romanov, A. E.; Pompe, W. J. Appl. Phys. 1995, 78, 1696-1706.

(4) Lymperakis, L.; Neugebauer, J.; Albrecht, M.; Remmele, T.; Strunk, H. P. Phys. Rev. Lett. 2004, 93, 196401.

(5) Chu, M. W.; Szafraniak, I.; Scholz, R.; Harnagea, C.; Hesse, D.; Alexe, M.; Gösele, U. Nat. Mater. 2004, 3, 87-90.

(6) Amalinckx, S. The Direct Observation of Dislocations. In Solid State Physics; Academic Press: 1964; Vol. 6.

(7) Miltat, J. E.; Bowen, D. K. J. J. Appl. Crystallogr. 1975, 8, 657669.

(8) Hÿtch, M. J.; Putaux, J. L.; Pénisson, J. M. Nature 2003, 423, 270-273.

(9) Lubk, A.; Rossell, M. D.; Seidel, J.; Chu, Y. H.; Ramesh, R.; Hÿtch, M. J.; Snoeck, E. Nano Lett. 2013, 13, 1410-1415.

(10) Simons, H.; King, A.; Ludwig, W.; Detlefs, C.; Pantleon, W.; Schmidt, S.; Stöhr, F.; Snigireva, I.; Snigirev, A.; Poulsen, H. F. Nat. Commun. 2015, 6, 6098.

(11) Simons, H.; Jakobsen, A. C.; Ahl, S. R.; Detlefs, C.; Poulsen, H. F. MRS Bull. 2016, 41, 454-459.

(12) Poulsen, H. F.; Jakobsen, A. C.; Simons, H.; Ahl, S. R.; Cook, P. K.; Detlefs, C. J. Appl. Crystallogr. 2017, 50, 1441-1456.

(13) Petroff, J. F.; Sauvage, M.; Riglet, P.; Hashizume, H. Philos. Mag. A 1980, 42, 319-338.

(14) Pietsch, U.; Holy, V.; Baumbach, T. High-resolution X-ray scattering: from thin films to lateral nanostructures 2004, 1 .

(15) Béa, H.; Bibes, M.; Sirena, M.; Herranz, G.; Bouzehouane, K.; Jacquet, E.; Fusil, S.; Paruch, P.; Dawber, M.; Contour, J. P.; Barthélémy, A. Appl. Phys. Lett. 2006, 88, 062502.

(16) Seidel, J.; Martin, L. W.; He, Q.; Zhan, Q.; Chu, Y. H.; Rother, A.; Hawkridge, M. E.; Maksymovych, P.; Yu, P.; Gajek, M.; Balke, N.; Kalinin, S. V.; Gemming, S.; Wang, F.; Catalan, G.; Scott, J. F.;
Spaldin, N. A.; Orenstein, J.; Ramesh, R. Nat. Mater. 2009, 8, 229234.

(17) Chu, K.; Jang, B. K.; Sung, J. H.; Shin, Y. A.; Lee, E. S.; Song, K.; Lee, J. H.; Woo, C. S.; Kim, S. J.; Choi, S. Y.; Koo, T. Y.; Kim, Y. H.; Oh, S. H.; Jo, M. H.; Yang, C.-H. Nat. Nanotechnol. 2015, 10, 972-979.

(18) Zeches, R. J.; Rossell, M. D.; Zhang, J. X.; Hatt, A. J.; He, Q.; Yang, C. H.; Kumar, A.; Wang, C. H.; Melville, A.; Adamo, C.; Sheng, G.; Chu, Y. H.; Ihlefeld, J. F.; Erni, R.; Ederer, R.; Gopalan, V.; Chen, L. Q.; Schlom, D. G.; Spaldin, N. A.; Martin, L. W.; Ramesh, R. Science 2009, 326, 977-980.

(19) Chu, Y. H.; Zhan, Q.; Martin, L. W.; Cruz, M. P.; Yang, P. L.; Pabst, G. W.; Zavaliche, F.; Yang, S. Y.; Zhang, J. X.; Chen, L. Q.; Schlom, D. G.; Lin, I. N.; Wu, T. B.; Ramesh, R. Adv. Mater. 2006, 18, 2307-2311.

(20) Jia, C. L.; Mi, S. B.; Urban, K.; Vrejoiu, I.; Alexe, M.; Hesse, D. Phys. Rev. Lett. 2009, 102, 117601.

(21) Sichel, R. J.; Grigoriev, A.; Do, D. H.; Baek, S. H.; Jang, H. W.; Folkman, C. M.; Eom, C. B.; Cai, Z.; Evans, P. G. Appl. Phys. Lett. 2010, 96, 051901.

(22) Xu, Y. B.; Tang, Y. L.; Zhu, Y. J.; Li, S.; Zhang, S. R.; Ma, X. L.; Liu, Y. Sci. Rep. 2016, 6, 35172.

(23) Liu, Y.; Tang, Y. L.; Zhu, Y. L.; Wang, W. Y.; Ma, X. L. Adv. Mater. Interfaces 2016, 3, 1600342.

(24) Khachaturyan, A. G. Theory of structural transformations in solids; Dover: New York, 1983.

(25) Liu, H.; Yang, P.; Yao, K.; Wang, J. Appl. Phys. Lett. 2010, 96, 012901.

(26) Suzuki, T.; Nishi, Y.; Fujimoto, M. Philos. Mag. A 1999, 79, 2461-2483.

(27) Lee, D.; Behera, R. K.; Wu, P.; Xu, H.; Li, Y. L.; Sinnott, S. B.; Phillpot, S. R.; Chen, L. Q.; Gopalan, V. Phys. Rev. B: Condens. Matter Mater. Phys. 2009, 80, 060102.

(28) Nye, J. F. Acta Metall. 1953, 1, 153-162.

(29) Kröner, E. Z. Eur. Phys. J. A 1955, 142, 463-475.

(30) Pantleon, W. Scr. Mater. 2008, 58, 994-997.

(31) Liu, G.; Zhang, Q.; Huang, H. H.; Munroe, P.; Nagarajan, V.; Simons, H.; Hong, Z.; Chen, L. Q. Adv. Mater. Interfaces 2016, 3, 1600444.

(32) Tagantsev, A. K. Phys. Rev. B: Condens. Matter Mater. Phys. 1986, 34, 5883.

(33) Catalan, G.; Lubk, A.; Vlooswijk, A. H. G.; Snoeck, E.; Magen, C.; Janssens, A.; Rispens, G.; Rijnders, G.; Blank, D. H. A.; Noheda, B. Nat. Mater. 2011, 10, 963-967.

(34) Biancoli, A.; Fancher, C. M.; Jones, J. L.; Damjanovic, D. Nat. Mater. 2015, 14, 224.

(35) Cao, W.; Cross, L. E. Phys. Rev. B: Condens. Matter Mater. Phys. 1991, 44, 5-12.

(36) Taylor, G. I. Proc. R. Soc. London, Ser. A 1934, 145, 362-387.

(37) Kogan, S. M. Sov. Phys. Solid State. 1964, 5, 2069.

(38) Indenbom, V. L.; Loginov, E. B.; Osipov, M. A. Sov. Phys. Crytallogr. 1991, 26, 656-658.

(39) Cheng, C. E.; Liu, H. J.; Dinelli, F.; Chen, Y. C.; Chang, C. S.; Chien, F. S. S.; Chu, Y. H. Sci. Rep. 2015, 5, 8091.

(40) Zubko, P.; Catalan, G.; Tagantsev, A. K. Annu. Rev. Mater. Res. 2013, 43, 387-421.

(41) Bratkovsky, A. M.; Levanyuk, A. P. Phys. Rev. B: Condens. Matter Mater. Phys. 2000, 61, 15042.

(42) Bednyakov, P. S.; Sluka, T.; Tagantsev, A. K.; Damjanovic, D.; Setter, N. Sci. Rep. 2015, 5, 15819.

(43) Horstemeyer, M. F. Integrated computation materials engineering (ICME) for metals: using multiscale modeling to invigorate engineering design with science 2012, 1. 